\title{
A sample of star forming regions triggered by cloud-cloud collision
}

\author{
Bei $\mathrm{Xin}^{1} \dagger$ and Jun-Jie Wang ${ }^{1}$ \\ ${ }^{1}$ National Astronomical Observatories, Chinese Academy of Sciences, Beijing, 100012, China. \\ e-mail:xp@bao.ac.cn and wangjj@bao.ac.cn
}

Collision between molecular clouds is considered an efficient mechanism to trigger cloud collapse to form stars. Various observations show that the process is taking place in the universe (Vallee 1995; Wang et al. 2004).

We conducted a large-sampling survey for the infrared sources, which are possibly regions of cloud-cloud collision, by making ${ }^{12} \mathrm{CO}(1-0),{ }^{13} \mathrm{CO}(1-0)$, and $\mathrm{C}^{18} \mathrm{O}(1-0)$ molecular line observations. We selected IRAS sources based on a molecular line survey in the Galaxy (Yang et al. 2002), and we picked up the ones whose ${ }^{12} \mathrm{CO}(1-0)$ molecular line is double-peaked or multi-peaked. Observations were made in November and December 2005 with the $13.7 \mathrm{~m}$ radio telescope at Delingha, the millimeter-wave radio observatory of Purple Mountain Observatory, in the $\mathrm{J}=1-0$ line of ${ }^{12} \mathrm{CO}$ at $115 \mathrm{GHz},{ }^{13} \mathrm{CO}$ at $110 \mathrm{GHz}$ and $\mathrm{C}^{18} \mathrm{O}$ at $110 \mathrm{GHz}$.

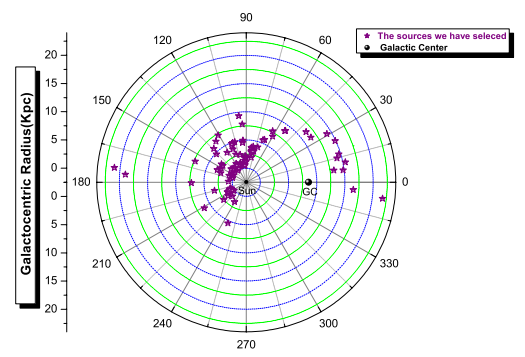

Figure 1: distribution of sources in our sample projected onto the Galactic plane.

We eliminated those whose optically thinner line peaks at the absorption part of the ${ }^{12} \mathrm{CO}$ (1-0) double line. They are possibly molecular clouds that are collapsing, and the double-peaked ${ }^{12} \mathrm{CO}$ (1-0) line is a possible sign of self-absorption (Zhou et al. 1993). We kept the sources whose molecular lines' features accord with the description of cloud-cloud collision, i.e. all the three molecular lines have two or more components at same velocity. Finally, we get rid of the possibility that the cloud is infalling, then built up a sample of those cold IRAS sources which are possibly cloud-cloud collision regions. A total of 214 sources were examined, and finally we got 135 of them according to cloud collision criterion. We have done some primary analysis of this data as well. Figure 1 shows the source distribution projected onto the Galactic plane. It can be seen that there are much more sources near the sun than those who are far away from us. More detailed research is carrying through now, and we hope this work can provide astronomers with useful information for their further studies.

\section{References}

Vallee, J.P. 1995, AJ 110, 2256

Wang, J.J., Chen, W.P., Miller, M., Qin, S.L.\& Wu, Y.F. 2004, ApJ 614, L105

Yang, J., Jiang, Z.B., Wang, M., Ju, B.G. \& Wang, H.C. 2002, ApJS 141, 157

Zhou, S., Evans, N.J., II, Koempe, C. \& Walmsley, C.M. 1993, ApJ 404, 232

$\dagger$ Present address: 20A Datun Road, Chaoyang District, Beijing, 100012, China. 